\title{
Parâmetros teóricos para elaboração de instrumentos pragmáticos de representação e organização da informação na Web: considerações preliminares sobre uma possível proposta metodológica ${ }^{1}$
}

\author{
Theoretical parameters for development of pragmatic tools of representation and \\ organization of information on the web: preliminary considerations on a possible \\ methodological proposal
}

\author{
Luciana de Souza Gracioso \\ Doutora em Ciência da Informação pelo IBICT/UFF. \\ Profa. Dra. do Departamento de Ciência da Informação/UFSCar \\ E-mail: luciana@ufscar.br
}

\section{Resumo}

\begin{abstract}
Muitos esforços têm sido feitos na busca de critérios conceituais e metodológicos para a sistematização de linguagens de interface para organização e recuperação de conteúdos em plataformas virtuais interativas. Neste sentido, um movimento desprovido de métodos universais a priori, se estabeleceu no cenário colaborativo da Web procurando indexar os conteúdos dinamicamente produzidos pelos usuários da rede: as folksonomias. Sob outra perspectiva existe, em ambientes sistematizados da informação, a validação e aplicação de vocabulários controlados como instrumentos de recuperação da informação. É no entroncamento destes dois caminhos que desenvolvemos esta pesquisa e iniciamos a discussão sobre uma proposta metodológica para construção de instrumento de recuperação da informação que considere na sua estrutura a linguagem cotidiana. $\mathrm{O}$ que se nomeia linguagem cotidiana aqui diz respeito a linguagem de busca (e ação) da informação e se difere, em certa medida, do conceito de linguagem natural enquanto linguagem que compõe textos. Selecionamos a CCS (Coordenadoria de Comunicação Social) da UFSCar (Universidade Federal de São Carlos) e o sistema desenvolvido por ela, o SACI (Sistema de Apoio de Comunicação Integrada) para análise do contexto de experimentação da metodologia proposta. Neste sistema selecionamos os clippings como produto informacional para análise das condições sobre a proposta de uma linguagem de indexação aberta. As iniciativas para constituição da referida proposta utilizam como parâmetro de aproximação e contradição as normas de Metodologia para construção de vocabulário controlado e Norma ANSI/ NISO Z39.19-2005. Outros projetos estão atrelados a esta pesquisa.
\end{abstract}

Palavras-chave: Folksonomia; Pragmática; vocabulário controlado.

\footnotetext{
${ }^{1}$ Este estudo é parte integrante do projeto de pesquisa "Proposta para identificação e aplicação de parâmetros teóricos e metodológicos na elaboração de instrumentos pragmáticos de representação e organização do conhecimento", financiado pelo CNPq. O projeto de pesquisa de iniciação científica "Proposta de elaboração de metodologia para construção de instrumento de recuperação da informação, a partir da linguagem cotidiana, em plataformas interativas (Web 2.0)", desenvolvida pela bolsista Jaqueline Birulio Peres, CNPq, no âmbito do projeto de pesquisa institucional de Gracioso (2009-2011) "A linguagem cotidiana como dispositivo de redes de informação: análises sobre as condições de significação das ações de informação científica e social mediadas por plataformas virtuais interativas" também está atrelado a esta pesquisa, além do projeto de extensão coordenado pela dra. Vera R. C Boccato intitulado: Organização e gestão da inovação em processos e produtos informacionais para a comunicação na UFSCar, vinculado ao Programa de Extensão coordenado pela dra. Cristina C. Ferraz.
}

InCID: R. Ci. Inf. e Doc., Ribeirão Preto, v. 1, n.1, p. 138-158, 2010. 


\begin{abstract}
Much effort has been done in order to find conceptual and methodological criteria to build a system of interface languages to organize and retrieve of contents in interactive virtual platforms. In this way a movement without the intention a priori to use universal methods was established in the collaborative scenario in the Web trying to index the contents dynamically produced both by people: the folksonomy. Besides, there is another information system perspective, in which the application and validation of controlled vocabulary is used as instrument information retrieval. Crossing these two perspectives that this research has been developed and a discussion is started about a methodological proposal to make a retrieval information instrument that considers in its structure the daily language. It is understood as daily language what is about the language and action to seek information and it is different, in a way, from the natural language concept as the language that composes text. It was selected the CCS (Coordenadoria de Comunicação Social) from UFSCar (Universidade Federal de São Carlos) and the system developed by CCS, o SACI (Sistema de Apoio de Comunicação Integrada) to analyze the context of experimentation of methodology proposed. In this system the Clipping was selected as information product to analyze the conditions for proposal to an open indexing language. The initiatives to build the proposal referred uses as approximation and contradiction parameters the rules of Methodology for the construction of controlled vocabulary and norm ANSI/ NISO Z39.19-2005. Other projects are evolved in this research.
\end{abstract}

Keywords: Folksonomy; Pragmatic; control vocabulary.

\title{
Introdução ao contexto pragmático de representação da informação
}

No ambiente sistematizado e institucionalizado de organização e recuperação da informação, que são representados em ampla escala pelas bases de dados, a ampliação das possibilidades de relações interdisciplinares e interconceituais entre as áreas, somadas à ampliação do uso das tecnologias de comunicação pela ciência, tem demandado uma reavaliação sobre a aplicação dos instrumentos de representação da informação. Sobre estas implicações González de Gomez (2004, p. 64) pondera que "no cada vez mais complexo sistema de ciência e tecnologia, encontram-se problemas para descrever e ainda organizar a produção contemporânea de conhecimentos científicos, à luz das categorias prévias de 'disciplina' e 'comunidade científica'”. Estas relações disciplinares e entre comunidades científicas tomam proporções cada vez maiores, intensificando o desafio de estruturação de modelos e instrumentos de intermediação informacional unívocos. Soma-se a isto a possibilidade de abertura destes sistemas para interferências cada vez mais frequentes de seus usuários, ora especializados, ou não. A imersão destes sistemas em plataformas abertas da Web agrega características dinâmicas e subjetivas ao desafio representacional. 
As diversidades das linguagens - verbais ou de imagens - e a heterologia dos conteúdos culturais parecem confinados a encontrar um termo de equivalência nos códigos homológicos das linguagens digitais. [...] Por um lado, desenha-se, em torno da questão da representação, um campo interdisciplinar que reúne, ao menos, as ciências do conhecimento, da linguagem, da informação e suas interfaces com as novas tecnologias. [...] A diversidade dos códigos de interpretações representacionais, assemelhados em sua base semiótica, serão agora pluralidades simultâneas e da mesma ordem: agregados de camadas de signos referenciando-se umas às outras, sem exemplares nem níveis privilegiados (GONZÁLEZ DE GOMEZ, 1993, p. 221).

O cenário citado antevê, no nosso entendimento, o ambiente interativo e colaborativo da Web, no que diz respeito às ações de uso social da linguagem para representar conteúdos tanto textuais, imagéticos, sonoros, e afins, que se entrecruzam na rede e que são utilizados do mesmo modo, sem sobreposições impostas. E esta estratégia sugerida no plano computacional, que permite a representação tanto a manifestação individual como coletiva na composição e na organização de conteúdos informacionais é que tem reforçado a necessidade de repensarmos as estruturas já solidificadas para instrumentalização dos processos de intermediação da informação.

Os esquemas de representação da informação pertencem à agenda de estudos da Ciência da Informação há décadas e autores, sob diferentes abordagens, já sugeriram a expansão sobre o uso de conceitos como descritores nos sistemas de representação da informação, que se aproximassem de seu contexto de uso. Atualmente a defesa sobre uma flexibilização dos vocabulários controlados tem, novamente, ganho corpus. Mais recentemente Guedes e Dias (2010) apresentam alguns desses estudos clássicos da Ciência da Informação, como o de Brown et al (1996), que foi analisado por Lancaster (1994) e que sugere um tratamento democrático da indexação em que, caso identificada a necessidade, os usuários acrescentariam aos registros os termos de sua própria escolha. Outros autores também apresentados por Guedes e Dias (2010), como Rafferty e Hidderley (1997), também apoiam um método de tratamento participativo da indexação. Em trabalhos anteriores, Gracioso (2008) apresenta algumas pesquisas que, ancoradas em alguns dos fundamentos da abordagem Pragmática para estudos da Ciência da Informação, também defendem esse mesmo propósito.

A Pragmática é uma abordagem da filosofia que, em sua essência, estuda o contexto de ações envolvidas no uso da linguagem e na produção do conhecimento. Muitos autores e muitas linhas são discriminadas e se confirmam na literatura como abordagem Pragmática. Nós preferimos por diferentes motivos nos orientar pelos indicadores dados por L. 
Wittgenstein (1889-1951), filósofo austríaco que tem como marco de sua produção pragmática a obra Investigações Filosóficas (publicada postumamente em 1953). O núcleo de sua abordagem é considerar que "a linguagem é o seu uso". E por isso, principalmente, consideramos o olhar deste autor um dos mais coerentes para iluminar os estudos sobre o uso de vocabulário na Web 2.0, que aqui chamamos de Web pragmática, para descrição e representação de conteúdos. Vale a ressalva que o conceito de Web pragmática, sugerido por Gracioso em 2007, tem sido considerado em diferentes frentes de estudo tanto nos EUA como na Europa. Em 2006 foi lançado o manifesto da Web pragmática no qual se estabelecem tanto os aportes teóricos pragmáticos estudados quanto as metodologias de construção de instrumentos de análise e avaliação das práticas sociais, comunicativas e interativas via internet, no contexto do que até então também se nomeia Web 2.0.

Blair (2003, 2006) é um dos autores da Ciência da Informação que faz algumas discussões sobre a aproximação da abordagem Pragmática para analisar os sistemas de organização e representação da informação. Discute principalmente a reconfiguração pragmática nos processos de representação temática, e os analisam como ação de descrição e de discriminação, tanto em relação aos conteúdos quanto aos usuários dos sistemas de informação. Blair não analisa os espaços virtuais abertos e interativos de informação e sim os sistematizados. $\mathrm{O}$ autor inclusive analisa as ações do sujeito frente ao sistema de informação a partir do que ele descreve como necessidade informacional e a partir de como ele discrimina o que lhe foi retornado pelo sistema, e considera que o modo de ser pragmático interfere diretamente nessas ações do sujeito. Os problemas relacionados às formas de descrição do buscador de informação sobre o que se quer buscar também precisaram ser discutidos, visto que, em certa medida, a equivalência das descrições entre o conteúdo do documento representado nos sistemas de informação e a necessidade do usuário que busca essas informações ocasionaria em recuperações precisas.

O contexto da descrição do conceito utilizado na pesquisa, nos buscadores abertos da Web, sofre a interferência da estatística dentre outros elementos. Há uma indicação lógica computacional que considera o termo mais buscado como "o termo preferido" para linkar a conteúdos e disponibilizá-los, isto acarreta em vários desencontros na recuperação precisa da informação. É importante que pesquisadores (buscadores de informações) tenham a dimensão da indeterminação da relação entre o que eles descreverão e o que, diante do que foi recuperado, terão de discriminar. Nesse ponto, observamos a formação de associações e cooperações sociais na Web (as redes sociais de informação), que seriam uma forma InCID: R. Ci. Inf. e Doc., Ribeirão Preto, v. 1, n.1, p. 138-158, 2010. 
distribuída de busca e discriminação de conteúdos, e a configuração das folksonomias seria um de seus resultados, ou produtos.

Outros autores, além de Blair, pensaram a Pragmática na perspectiva informacional. Dentre esses estudos, podemos destacar o de B. Frohmann (1990). O autor desenvolve um estudo mais direcionado aos princípios apresentados na obra Investigações Filosóficas (1953) de L. Wittgenstein e os analisa à luz da Ciência da Informação. Considera que, para fins de indexação, não bastaria a compreensão dos processos cognitivos individuais, mesmo porque esses não existiriam isoladamente. $\mathrm{O}$ mérito da proposta apresentada nesta obra estaria em demonstrar que a possibilidade de compreensão da forma de pensar do homem está relacionada à sua forma de viver, de se expressar e de se fazer entender pelos membros de sua comunidade. Essas convenções sociais delimitam a forma de pensar, assim, esse se aprenderia apenas socialmente, com a prática. O pensar seria uma prática social. O autor acredita que somente considerando esses princípios o indexador seria capaz de representar conteúdo de maneira a atribuir-lhes relevância para seus potenciais usuários (FROHMANN, 1990).

Ainda na ótica da Pragmática proposta pelo filósofo L. Wittgenstein, W. Nedobity (1989) procura avaliar os diferentes métodos de investigação do significado da linguagem no contexto da terminologia. É importante mencionar que Wüster, analisado por Nedobity, foi o precursor da Teoria da Terminologia, atualmente estudada pela Ciência da Informação como um dos possíveis caminhos para compreensão da construção de sentido do conceito, que busca aplicar os fundamentos da lógica e da epistemologia para resolver os problemas de comunicação de assuntos entre os especialistas. Em síntese, sua tese procura orientar o uso do conceito somente no contexto (previamente delimitado) que lhe é mais apropriado. Nedobity (1989) atribui o contraste desta à teoria de Wittgenstein, na medida em que o princípio da Terminologia proposta por Wüster estaria em utilizar uma regra central para a compreensão de uma linguagem especializada, e a definição do conceito se daria a partir do uso desta regra. Wüster analisaria o significado como sendo construído a partir da relação entre conceitos. Já para Wittgenstein, não haveria possibilidade de prever, estabelecer e reutilizar essas relações conceituais, tendo em vista que elas se constituem exclusivamente em contextos específicos de forma de vida em contexto de uso da linguagem.

Para justificar um pouco mais a validez de se desenvolver estudos teóricos pragmáticos por meio da Ciência da Informação, mencionamos o trabalho de Hjorland (1998), que discute suas implicações semânticas nos processos de recuperação da informação. O autor 
considera que as discussões da segunda fase de Wittgenstein, feitas na obra Investigações filosóficas, a partir dos jogos de linguagem, poderiam sustentar algumas das implicações inerentes aos processos de recuperação da informação por considerar não haver limites entre as observações, os conceitos e as teorias. Todos esses itens estão inseridos em um contexto social e histórico, que precisa ser analisado, e essas condições de construção de significado são discutidas (porém não operacionalizadas) por Wittgenstein. Hjorland afirma que os sistemas de recuperação de informação requerem regras operacionais para sua consolidação, mas mesmo não havendo, inicialmente, uma aplicação direta dos princípios de construção de significado propostos por Wittgenstein, eles precisariam ser considerados durante os processos de desenvolvimento e aperfeiçoamento desses sistemas, pois somente assim, acredita o autor, seria possível providenciar conteúdos semânticos recuperáveis precisamente (HJORLAND, 1998).

No Brasil, o trabalho de Novelino (1998) também defende a necessidade de convalidarmos questões pragmáticas para subsidiar a organização de conteúdos, propondo que sejam construídas linguagens de "transferência da informação", e não simplesmente de representação temática de conteúdos. Na transferência, haveria a reconstrução e a contextualização dos conceitos e dos significados utilizados na construção dos conteúdos na medida em que estes fossem representados também pelas formas de uso como foram buscados. Para a autora, as linguagens de recuperação da informação devem buscar o significado dos conceitos em seus contextos de produção e de uso.

Tanto os trabalhos de D. Blair (2003, 2006), B. Frohmann (1990) como os de Nedobity (1989), Hjorland (1998) e Novelino (1998), que apresentamos brevemente, discutem as implicações da necessidade e as consequências da adoção da perspectiva pragmática no contexto da representação e da recuperação da informação em sistemas de informação. Mas o nosso intuito é de pensarmos, na esfera das ações informacionais, em espaços de informação abertos, interativos e dinâmicos.

Ainda no cenário nacional, Kobashi publica em 2007 um artigo em que discute os fundamentos semânticos e pragmáticos da construção de instrumentos de representação da informação, no entanto, sem se ater exclusivamente às abordagens wittgensteinianas. Kobashi (2007, p.2) define a "pragmática linguística como abordagem que estuda os fatores que regem as escolhas linguísticas na interação social" e indica que a relação da pragmática com as linguagens documentárias já fora acenada por Hutchins em 1975. Kobashi traça um contexto 
de estudo pragmático da linguagem relacionado às ações de representação documentária. Analisa o estabelecimento do significado do símbolo por convenção dentro de um paradigma da comunicabilidade da linguagem, de acordo com as abordagens de C. Peirce e o uso da língua como uma atividade social movida pela necessidade de comunicação, sugerida por M. Bakhtin e estudado em Cintra (2002) no plano da análise do discurso da sociolinguística e da socioterminologia. A linguagem seria de natureza essencialmente dialógica - sociointerativa e, de acordo com nossos pressupostos, esta seria também uma característica essencial das folksonomias. Wittgenstein, assim como Habermas e Apel são autores pragmáticos citados por Kobashi e estão relacionados aos estudos da informação que consideram o consenso sobre o significado alcançado intersubjetivamente - perspectiva que adotamos inclusive. A autora considera a abordagem Pragmática refletida no plano da interação entre usuário e dispositivo pensando em uma indexação para a pergunta que este faz e diante disto pondera que "a informação participa de diferentes estruturas de significação, o que motiva a reflexão permanente sobre os métodos de elaborar linguagens apropriadas para os diferentes contextos e seus públicos”. (KOBASHI, 2007, p. 2).

Lara (2008) estuda a Pragmática na Ciência da Informação relacionando aspectos da linguística documentária com as discussões de Hjorland e Capurro pensando a relação do conceito de informação como construção simbólica e sua relação nas comunidades discursivas, a construção das mensagens documentárias e a integração das categorias de recepção nos processos de organização da informação para acesso.

Análises pautadas na Teoria da ação comunicativa de Habermas (1981) sugerem a ampliação e a flexibilização do controle terminológico nos sistemas de informação, como as desenvolvidas por Benoît $(2001,2007)$. O autor discute a competência comunicativa aplicada e reconstruída nas ações de busca informacional sistêmica, inclusive desenvolve testes em sistemas informacionais e, somados esses a análises de outros estudos, conclui que a aproximação dos critérios de significação construídos entre "humanos" é difícil de ser apropriada (a priori) completamente por sistemas operacionais, mesmo quando esses estão voltados para interação. As sugestões do autor em relação aos sistemas de informação voltam-se, então, à análise de questões de ordem filosófica antes de sua estruturação, para que interfaces educativas sejam elaboradas e canais de comunicação, abertos. A partir do prelúdio à interação, esses sistemas poderiam se constituir comunicativamente, e seus conteúdos passariam a ser validados, a partir de seu uso, pelas pretensões de validade comunicativa (propostos por Habermas). Sem essa abertura, os sistemas de informação ficariam restritos às ações estratégicas, sem promover a significação, InCID: R. Ci. Inf. e Doc., Ribeirão Preto, v. 1, n.1, p. 138-158, 2010. 
permitindo somente a receptividade de informações. Outras aproximações da abordagem Pragmática no âmbito dos sistemas de informação têm se firmado na linha da LAP - Language Action Perspective, no entanto, sem se aterem a questões de vocabulários especificamente.

Os desafios latentes relacionados a representação da informação no plano reconfigurado das ações de comunicação na Web foram o objeto de boa parte das pesquisas apresentadas na International ISKO Conference de 2010. Nesta edição, a conferência teve como proposta reunir trabalhos que discutissem os paradigmas e os sistemas conceituais na organização do conhecimento. Como eixo conceitual, considera a nova situação global da interconectividade dos significados do conhecimento configurada a partir da diversidade de tradições e pontos de vista específicos (culturais, disciplinares e teóricos) que se constituem dentro deste quadro global, considerando as implicações sobre a antecipação das demandas para a busca de conhecimento. Neste universo de pesquisa, destacamos o trabalho de Dousa, que discute a relação do pragmatismo clássico e o neo pragmatismo de R. Rorty relacionado à organização do conhecimento. Na defesa da abordagem Pragmática para as ações de informação, também estão os trabalhos de Hjorland e Gracioso. Especificamente, analisaram a relação da linguagem natural com a organização do conhecimento nos trabalhos de Lykke et al, que discutiu o uso de componentes semânticos para representar e pesquisar documentos de domínios específicos. Já Moura e Assis analisaram as redes sociais, as linguagens de indexação e a organização do conhecimento no plano da semiótica, e Vakkari, a configuração de tesauros específicos e gerais que abranjam os vocabulários de leigos no contexto de saúde, nutrição e serviço social. Nesta linha Priss e Old, estudam o uso da linguagem natural em tesauros a partir do conceito de vizinhança entre sistemas. O trabalho de Huvila argumenta sobre as condições dos julgamentos estéticos representados nas folksonomias como critério para organização do conhecimento, e Smiraglia analisa os aspectos perceptivos e afetivos envolvidos no uso das tags para representar informações. Nessa linha foi apresentado o trabalho de Abreu, que analisa os gêneros, as atitudes e os afetos que fazem com que usuários definam o que é "legal" de ser representado na rede. Esses trabalhos, de modo geral, são indicativos de que há uma agenda emergente de pesquisas sobre as relações pragmáticas que são inerentes a organização do conhecimento na Web.

Considerando fundamentalmente as premissas wittgensteiniana e habermasiana sobre as implicações das ações comunicativas e informacionais, destacamos a pesquisa de Allowood e Lind (2008), que vinculam a Pragmática ao contexto interativo da Web. Em seu texto Making the web more pragmatic (2008), os autores utilizam os conceitos, ou ainda, as categorias de "social activity", "communicative act", "sequences of communicative acts" ou "exchange InCID: R. Ci. Inf. e Doc., Ribeirão Preto, v. 1, n.1, p. 138-158, 2010. 
types", "communicative feedback" e "turn management" para analisar serviços interativos da Web, como a Wikipedia.

Por outro viés de análise sobre as ações de organização e de representação da informação, a Ciência da Informação, no domínio das instituições e sistemas de informação, se apoia em garantias classicamente validadas para estabelecer suas diretrizes. Dentre elas destacaremos aquelas que podem alicerçar nossas escolhas metodológicas, como a garantia de usuário, que permite que os termos que serão selecionados para um tesauro estejam de acordo com os utilizados no ato da busca da informação, pelo usuário. "É importante acatar o vocabulário dos usuários e através dele conduzir as requisições que eles fazem aos descritores de um vocabulário mais especializado." Para os usuários, "é sempre útil incluir termos nos quais nunca poderiam pensar, mas para os quais poderiam ser dirigidos com a finalidade de melhorar os pedidos, em sua procura" dizem Moreira e Moura (2006). Svenonius (2000 apud MOREIRA; MOURA, 2006), “[...] considera que encontrar a linha de uso comum entre os termos nem sempre é o alvo principal para identificar os conceitos nos quais um usuário está interessado, mas é fundamental para dar nome a estes conceitos." Historicamente Moreira e Moura recuperam o trabalho de Gilchrist (1971) que tratou a garantia de uso, "como uma força paralela de pensamento na qual o leitor, como usuário e frequentemente criador de literatura, deveria ser empregado como uma fonte para se conhecer a linguagem mais apropriada para a indexação".

A essas garantias de uso, aproximamos os estudos de Beghtol (1986), não necessariamente voltada a questões computacionais, e o conceito de garantias culturais. A garantia cultural reforçaria que o estabelecimento de relações semânticas nos instrumentos de representação temática dependeria do contexto cultural na qual elas são utilizadas pelos pesquisadores. Contudo, no momento em que elementos culturais passam a ser garantias para representação do conhecimento, implicações éticas igualmente demandam por atenção. Guimarães et al. (2005) e Pinho (2006) são alguns dos autores que analisam essas implicações, e Gracioso em 2008 também sistematiza parte dessas discussões. Moura e Assis (2010) recuperam os estudos sobre as garantias relacionadas à organização do conhecimento pensando-as no contexto Web. Mas as indicações sobre a necessidade da busca pelo variável como critério de validação para organização do conhecimento, foram sinalizadas por González de Gomez, já em 1993, e continuam sendo pesquisadas pela autora, no contexto das abordagens da obra de J. Habermas (1929-). 
$\mathrm{Na}$ busca de parâmetros para estabelecer garantias de representação neste cenário tecnológico atual, Begthol sugere, ao analisar as bibliografias sobre classificação bibliográfica, o termo "hospitalidade". Tal termo se refere à capacidade de uma notação classificatória incluir novos conceitos relacionando-os semanticamente e sintaticamente aos já existentes. Beghtol (2002) recomenda que este conceito possa ser apropriado no contexto atual da representação, considerando que não sejam apenas incluídos novos conceitos as representações, mas que estes sejam acompanhados de elementos que representem o seu contexto de uso. O conceito sugerido pela autora para designar essa prática seria o de "hospitalidade cultural". Segundo Beghtol (2002), os sistemas de representação e de organização do conhecimento, para serem éticos no sentido de não privilegiarem ou excluírem contextos culturais, precisariam se sustentar em princípios éticos universais, ampliando as possibilidades de busca informacional do sujeito frente aos conteúdos disponíveis via rede.

Nesta pesquisa, sugere-se uma possível garantia pragmática para inserção de novos termos em instrumentos de intermediação temática desenvolvidos para ambientes de informação que estão atrelados à Web. Esta garantia seria a soma entre a garantia semântica e de uso, ou cultural, proposta por Begthol, em que se vincularia ao termo sugerido pelo usuário para inserção no vocabulário a descrição sobre o uso que faz deste conceito em seu contexto situacional de busca de informação. A ideia é de aproximar diferentes significações estabelecidas por diferentes usuários para um mesmo conceito e, na medida do possível, que este conceito, atrelado a sua significação, possa ser relacionado a conteúdos que de fato subjetivam este mesmo entendimento.

Mais pontualmente, retomamos que o trabalho que se apresenta aqui, ainda em desenvolvimento, tem o desafio de delimitar alguns preceitos para uma proposta metodológica para construção de instrumento de recuperação da informação, incorporando a linguagem cotidiana (linguagem de busca do usuário), em plataformas interativas virtuais. Como espaço, ou laboratório de observação e atuação, se estuda o ambiente CCS, pela natureza interativa adotada na construção de parte dos conteúdos que produz e vincula, pelo hibridismo entre conhecimentos científicos e sociais que lida, pela natureza hipermidiática de seus produtos informacionais e pela heterogeneidade de seus pólos de usuários. 


\section{Coordenadoria de Comunicação Social como espaço de interação e experimentação}

A gestão da Coordenadoria de Comunicação Social (CCS) da UFSCar, desde 2001, tem como foco a reestruturação e consolidação de troca de informações e comunicação da Universidade, interligando setores e departamentos que apoiam a iniciativa de propagação de conhecimento, dentre eles a pró-reitoria de extensão (rádio e TV), o sistema de bibliotecas, a editora universitária, entre outros. Seguindo o princípio de comunicação integrada e tomando diferentes frentes, a CCS busca a estruturação de produtos que viabilizem o cumprimento das demandas e expectativas da comunidade interna e externa da universidade, e se intitula enquanto espaço de experimentação de novas linguagens, formatos e meios de estrutura tecnológica, considerando possibilidades de formação formal e informal de cidadania.

Para facilitar a disponibilização de materiais registrados em suportes digitais e para a organização e controle do fluxo de informações, a CCS desenvolveu o SACI (Sistema de Apoio a Comunicação Integrada). Este software é open source e foi editado inicialmente para o uso em centros de comunicação social de universidades federais para disponibilização de notícias e produtos artísticos, científicos e culturais considerando sua respectiva armazenagem, recepção, produção e disseminação otimizada em plataformas digitais. Ao mesmo tempo, este software permite que seus alimentadores e seus usuários tenham sua ferramenta de trabalho, pesquisa e interação à disposição em qualquer espaço e em qualquer tempo. O SACI foi desenvolvido para oferecer uma otimização do trabalho da CCS na UFSCar e uma consolidação entre diferentes públicos e processos históricos dentro da universidade. Alguns estudos gerados e geradores deste Sistema foram feitos por Bermudez (2005), Botelho e Bela (2006), Cicillini e Francisco (2007), Deodato e Botelho (2004), Maia, Bassolini e Betolni (2008).

Um dos produtos informacionais metodologicamente mais explorados pela CCS é o clipping, que resulta do procedimento de selecionar notícias em jornais, revistas, sites ou qualquer outro meio de comunicação que tenham o tema pertinente em dado contexto, atividade que nos remete, inclusive, a nossas Hemerotecas, classicamente desenvolvidas nos espaços das bibliotecas. No caso da CCS, a política sobre os clippings está voltada a sistematizar e disponibilizar notícias sobre a própria Universidade tanto para seu uso interno como para que a sociedade tenha acesso sobre a notícia relacionada a instituição. A produção e organização dos clippings são feitas pela CCS desde 1980. Há um projeto de digitalização e indexação de todo acervo. No entanto somente os clippings de 2008 em diante estão InCID: R. Ci. Inf. e Doc., Ribeirão Preto, v. 1, n.1, p. 138-158, 2010. 
disponíveis digitalmente para busca e uso. Há uma lista com aproximadamente 8.000 descritores organizados alfabeticamente, que são utilizados como fonte de indexação do material, por estagiários do curso de Biblioteconomia e Ciência da Informação contratados para tal fim, mas não há nenhum controle sintático ou semântico sobre isso. Atualmente, a recuperação dos clippings é feita sem consulta a qualquer linguagem controlada. O que se recupera são materiais que foram indexados a partir de uma lista alfabética de conceitos, escolhidos a partir do conteúdo dos documentos (linguagem natural segundo Lancaster) e das percepções do estagiário indexador. Não há indexação automática de conceitos, e não foi feito nenhum mapeamento sobre os usuários e usos destes conteúdos relacionado às suas ações de busca.

\section{Aproximações e distanciamentos conceituais e metodológicos: resultados iniciais}

Optou-se em descrever mais detalhadamente os conceitos e as metodologias de construção de vocabulários controlados em sistemas de informação neste momento para sistematizar o encadeamento da apresentação de alguns resultados iniciais. Antes valeria ressaltar que, embora já tenham sido feitas muitas iniciativas de tratamento, sistematização, ou ainda compreensão das folksonomias e do comportamento das Tags como recursos de recuperação da informação em ambientes abertos e virtuais da informação, não se diagnosticou nenhuma construção metodológica aplicada a este ambiente puramente Web, mesmo porque qualquer iniciativa nesse sentido seria paradoxal. Pelo menos não de modo relacionado às metodologias vigentes de construção de vocabulário controlado desenvolvidos pela Biblioteconomia e Ciência da Informação. No entanto consideráveis trabalhos têm sido desenvolvidos por esta Ciência no trato desta reconfiguração sobre o tratamento da informação, no quais destacamos os estudos que mencionamos anteriormente. Seguimos assim, apresentando e aproximando às metodologias de construção de vocabulário controlado as possibilidades de representação permitidas pelas folksonomias, ainda em caráter exploratório e experimental.

As linhas gerais de construção e formatação de vocabulários controlados são apresentadas pela organização norte-americana National Information Standards Organization (2005). Mais especificamente sobre as regras de produção desses vocabulários (onde se incluem os tesauros, as taxonomias, as listas de sinônimos), a norma dita padrões para estabelecimento de associação, equivalência e hierarquia no vocabulário. Recentemente, esta

InCID: R. Ci. Inf. e Doc., Ribeirão Preto, v. 1, n.1, p. 138-158, 2010. 
mesma norma - ANSI/NISO Z39.19-2005 incluiu em seu escopo metodológico as especificidades propostas pelo Classification Research Group - CRG e a análise facetada proposta por Ranganathan mais voltada para os tesauros. Elementos de interoperabilidade entre linguagens também começam a se delimitar. Mas o que nos interessa nesta pesquisa é o conjunto de tratamentos sugeridos ao termo no processo de construção do vocabulário controlado para uso em sistemas de informação, e que foram apresentados inicialmente na versão de 2003 da referida norma. No entanto, são nos trabalhos desenvolvidos e apresentados no site de Biblioteconomia, Informação e Tecnologia da Informação - BITI (CAMPOS, GOMES, MOTTA, 2004) que encontramos mais claramente os elementos que analisaremos. A análise desses padrões e normas tem sido feita em diferentes contextos de estudo. Em síntese, um vocabulário controlado, tem os seguintes elementos: delimitação de campo de assunto, levantamento de descritores sobre o assunto, categorização dos descritores, estabelecimento de relações entre descritores em cada categoria - relação de equivalência, hierárquica e associativa e notas de escopo (descrição sobre os descritores). A seguir apresentamos alguns desses procedimentos que são propostos na configuração de vocabulários controlados e traçamos as aproximações e as não aproximações possíveis relacionadas às folksonomias: 


\begin{tabular}{|c|c|}
\hline $\begin{array}{l}\text { Metodologia para construção de } \\
\text { vocabulários controlados }\end{array}$ & Folksonomias \\
\hline $\begin{array}{l}\text { Planejamento } \\
\text { - delimitação da área do assunto que será } \\
\text { representado pelo vocabulário; } \\
\text { - definição de público-alvo do } \\
\text { vocabulário; } \\
\text { - procedimentos de manutenção. }\end{array}$ & $\begin{array}{l}\text { Planejamento } \\
\text { - não há delimitação de área de assunto a priori. As } \\
\text { folksonomias podem representar assuntos delimitados } \\
\text { em campos específicos do conhecimento e podem } \\
\text { representar assuntos diversos não classificados e } \\
\text { categorizados em nenhum campo específico; } \\
\text { - não há possibilidade de previsão de público alvo tanto } \\
\text { em relação aos construtores da folksonomia, quando em } \\
\text { relação aos seus consultores; } \\
\text { - a manutenção da folksonomia é feita } \\
\text { concomitantemente ao seu uso, sem políticas definidas. }\end{array}$ \\
\hline $\begin{array}{l}\text { Levantamento do vocabulário } \\
\text { - selecionar os termos representativos do } \\
\text { assunto e defini-los de acordo com a } \\
\text { natureza do assunto. }\end{array}$ & $\begin{array}{l}\text { Levantamento do vocabulário } \\
\text { - a seleção dos termos representativos não se vincula } \\
\text { exclusivamente ao conteúdo do documento. A } \\
\text { representatividade dos termos está atrelada ao usuário e } \\
\text { criador da folksonomia. A definição dos termos não é } \\
\text { feita de modo unívoco. Diferentes descrições e } \\
\text { significados podem ser relacionados a um mesmo } \\
\text { conceito. }\end{array}$ \\
\hline $\begin{array}{l}\text { Organização dos conceitos } \\
\text { - agrupar termos de mesma natureza em } \\
\text { categorias ou facetas. }\end{array}$ & $\begin{array}{l}\text { Organização dos conceitos } \\
\text { - os termos são agrupados de acordo com a maior } \\
\text { frequência de seu uso na marcação de conteúdos. }\end{array}$ \\
\hline $\begin{array}{l}\text { Apresentação final } \\
\text { - listagens alfabéticas e visualizações } \\
\text { gráficas. }\end{array}$ & $\begin{array}{l}\text { Apresentação final } \\
\text { - nuvens de tags (conceitos) destacados graficamente a } \\
\text { partir da quantidade de seu uso. }\end{array}$ \\
\hline $\begin{array}{l}\text { Critérios de avaliação } \\
\text { - índices de precisão e revocação. }\end{array}$ & $\begin{array}{l}\text { Critérios de avaliação } \\
\text { - índices de uso, sem métodos definidos. }\end{array}$ \\
\hline
\end{tabular}

Quadro 1: Análise comparada entre vocabulário controlado e folksonomia.

Outra análise comparada sobre as metodologias de construção de vocabulários controlados e as Folksonomias consiste nos conceitos de Descritor e Notas de escopo, dentre outros, definidos pela Norma Americana ANSI/NISO Z39.19-2005. 


\begin{tabular}{|c|c|}
\hline $\begin{array}{c}\text { Metodologia para construção de } \\
\text { vocabulário controlado }\end{array}$ & Folksonomias \\
\hline $\begin{array}{l}\text { Descritor } \\
\text { Cada descritor incluído em um vocabulário } \\
\text { controlado representa um conceito único (ou } \\
\text { unidade de ideia). Um conceito pode ser } \\
\text { expresso por termo de única palavra ou por um } \\
\text { termo com múltiplas palavras. O escopo dos } \\
\text { descritores é restringido para selecionados } \\
\text { significados em um domínio do vocabulário } \\
\text { controlado. Cada descritor pode ser formulado } \\
\text { em tal modo que convir o escopo intencionado } \\
\text { para cada usuário do vocabulário controlado. }\end{array}$ & $\begin{array}{l}\text { Descritor } \\
\text { O descritor (Tag) em uma folksonomia não } \\
\text { representa um conceito único. Sua descrição e } \\
\text { associação com os conteúdos que irá representar } \\
\text { são estabelecidas na ação de seu uso como } \\
\text { descritor. A indicação da relação entre descritor } \\
\text { (Tag) e conteúdo será estabelecida a partir do } \\
\text { significado dado ao descritor, pelo usuário- } \\
\text { indexador. }\end{array}$ \\
\hline Notas de escopo & Notas de escopo \\
\hline $\begin{array}{l}\text { As notas de escopo são usadas para restringir } \\
\text { ou expandir a aplicação de um descritor, para } \\
\text { distinguir entre descritores que têm } \\
\text { significados sobrepostos na linguagem natural, } \\
\text { ou para prover conselho no uso de outro termo } \\
\text { para o indexador e o pesquisador. Uma nota de } \\
\text { escopo deve situar o significado escolhido de } \\
\text { um descritor; ele pode também indicar outros } \\
\text { significados que são reconhecidos em } \\
\text { linguagem natural, mas que foram } \\
\text { deliberadamente excluídos do vocabulário } \\
\text { controlado. Uma nota de escopo não é uma } \\
\text { parte de um descritor. Uma nota de escopo } \\
\text { pode ser fornecida para cada descritor. }\end{array}$ & $\begin{array}{l}\text { Não há Notas de escopo em folksonomias. O } \\
\text { significado do conceito (Tag) indicado para a } \\
\text { indexação não é fixo, não é estabelecido a partir } \\
\text { do conteúdo do documento, não é estabelecido } \\
\text { pelo indexador a posteriori. O significado do } \\
\text { conceito utilizado no taggeamento é variável ao } \\
\text { entendimento de cada taggeador. A inclusão de } \\
\text { notas de escopo em Tags pode situar o } \\
\text { significado escolhido de um descritor e pode } \\
\text { indicar outros resultados que são reconhecidos } \\
\text { em linguagem cotidiana e não poderão ser } \\
\text { excluídos. Várias notas de escopo poderão ser } \\
\text { fornecidas para um mesmo descritor. }\end{array}$ \\
\hline $\begin{array}{l}\text { Tipos de relacionamentos } \\
\text { - Equivalência; } \\
\text { - Hierárquico; } \\
\text { - Associativo. }\end{array}$ & $\begin{array}{l}\text { Tipos de relacionamento } \\
\text { As folksonomias não preveem sistematizações } \\
\text { hierárquicas e associativas entre as Tags. A } \\
\text { indicação de Termo preferido é estabelecida a } \\
\text { partir da predominância do uso do conceito na } \\
\text { indexação (Taggeamento). }\end{array}$ \\
\hline
\end{tabular}

Quadro 2: Análise conceitual comparada entre vocabulário controlado e folksonomia.

Diante das aproximações e não aproximações possíveis entre as metodologias vigentes sobre vocabulário controlado e as Folksonomias, tornam-se claras as diferenças entre ambas. No entanto, elas têm a mesma função - a de representar, através do uso de conceitos (controlados ou não) os conhecimentos produzidos e disponibilizados em sistemas de informação (no caso de vocabulários controlados) ou em ambientes abertos da Web (como as Folksonomias). Considerando a existência de espaços híbridos de controle e abertura de produção e uso da informação, como é o caso das plataformas interativas (Web pragmática), a 
proposta de uma linguagem híbrida, no que se refere aos níveis de controle, parece necessária. Sobre as condições pragmáticas e dinâmicas de significação, convém nos apoiarmos na definição, no contexto desta pesquisa, à noção de conceito proposta por Hjorland em 2009 e reafirmada em 2010 (HJORLAND, 2010), de que "conceitos são dinamicamente construídos e seus significados, que classificam o mundo acordando interesses e teorias, são coletivamente negociados"2 (HJORLAND, 2009, p. 1522-1523).

No caso da CCS, de seus produtos informacionais e de seu público alvo, esse hibridismo precisaria ser previsto em qualquer linguagem que se propõe a representar e recuperar as informações produzidas. A necessidade de mesclar elementos metodológicos de construção de vocabulário controlado e folksonomias caberia em espaços parecidos ao da CCS. Para alguns sistemas, se aplicam unicamente os vocabulários controlados e, para outros, somente as Folksonomias. A questão é que cada vez mais nos deparamos com o hibridismo potencializado pela/na Web, o que nos instiga a procurar ainda mais mecanismos possíveis de aproximação entre essas linguagens de naturezas distintas, mas com funções comuns.

\section{Proposta inicial de elaboração de metodologia para construção de instrumento de recuperação da informação, a partir da linguagem cotidiana, em plataformas interativas - análise dos clippings, CCS}

A partir da lista de descritores gerada pela indexação não controlada dos Clippings na CCS, sugere-se:

1 Elaboração inicial de taxonomias a partir dos conceitos já utilizados (projeto em desenvolvimento como atividade de extensão da UFSCar);

a. Categorização da lista de termos e estabelecimento de relacionamentos entre eles.

i. Inclusão dos termos em categorias conceituais sugeridas

ii. Estabelecimento de relações entre os termos dentro das categorias

iii. Delimitação de Termos preferido para uso na indexação

iv. Delimitação de Nota de escopo do termo

\footnotetext{
${ }^{2}$ Tradução livre de Concepts are dynamically constructed and collectively negotiated meanings that classify the world according to interests and theories (HJORLAND, 2009, p. 1522-1523).

InCID: R. Ci. Inf. e Doc., Ribeirão Preto, v. 1, n.1, p. 138-158, 2010.
} 
2 Orientação sobre o ambiente computacional SACI para inserção da taxonomia considerando as seguintes aberturas para inclusão de conceitos e suas definições:

a. Inclusão de termos utilizados pelos usuários na busca por conteúdo como conceito de indexação, para posterior ajuste em categorias e estabelecimento de relacionamentos.

b. Inclusão de número ilimitado de campos de Nota de escopo para descrição do conceito que será feita, também, pelo usuário - esta descrição pragmática poderá se dar tanto sobre os conceitos inéditos que este usuário utiliza na busca de informação (potencial descritor) quanto sobre o conceito já existente na taxonomia, caso se opte por fazer a busca utilizando o vocabulário controlado.

A verificação da abertura do SACI para empenho destas atribuições do vocabulário já foi feita junto aos responsáveis pelo sistema, que terá sua versão 3.0 em 2011. Como continuidade desta pesquisa, pretende-se ainda avaliar os índices de precisão de recuperação dos clippings a partir do controle terminológico vigente utilizado na indexação do material, apurar e sistematizar a viabilidade de aplicação de uma plataforma para sistematização de vocabulário a partir da metodologia sugerida, desenvolver teste de aplicação da metodologia em corpus conceitual específico, estipular critérios para testar a aceitação de usuários na interação com o vocabulário, aplicar a metodologia em questão com a participação de uma amostra de usuários e estabelecer critérios para avaliação da precisão da recuperação da informação na configuração proposta.

Com a inserção destas ações iniciais de abertura para entrada de conceitos e descrições cotidianas em linguagens controladas, como no caso da que está em desenvolvimento para a indexação dos clippings na CCS, visualiza-se um pequeno passo para a construção de um procedimento flexível e híbrido voltado ao uso de conceitos para representação e recuperação da informação em ambientes interativos informacionais da Web. De qualquer modo, muito ainda se tem a estudar sobre a necessidade e as reais possibilidades dessas aproximações e propostas metodológicas que sinalizamos em caráter experimental. Evidentemente, o desenvolvimento, a aplicabilidade e principalmente o sucesso nas ações de busca e recuperação da informação, diante do que propomos neste momento, precisarão ser diagnosticados e analisados, e neste sentido a continuidade de pesquisas sobre essas implicações se faz mais do que necessária, senão urgente!

InCID: R. Ci. Inf. e Doc., Ribeirão Preto, v. 1, n.1, p. 138-158, 2010. 


\section{Referências}

ALLOWOOD, J.; LIND, M. Making the web more pragmatic: exploring the potential of some pragmatic concepts for is research and development. In: INAUGURAL MEETING OF AIS SPECIAL INTEREST GROUP ON PRAGMATIST IS RESEARCH, 2008, Paris. Proceedings...Uppsala: Uppsala University, 2008. p. 67-72.

BEGHTOL, C. Semantic validity: concepts of warrant in bibliographic classification systems. Library Resources \& Technical Services, v. 30, n. 2, p. 109-25, 1986.

A proposed ethical warrant for global knowledge representation and organization systems. Journal of Documentation, London, v.58, n.5, p. 507-32, 2002.

BENOÎT, G. Critical theory as a foundation for pragmatic information systems design. Information research, v. 6, n. 2, 2001. Disponível em: http://informationr.net/ir/62/paper98.html. Acesso em: 25 jul. 2008

Critical theory and the legitimation of library and information science.

Information research, v.12, n. 4, 2007. Disponível em: http://informationr.net/ir/124/colis/colis30.html. Acesso em: 25 jul. 2008.

BERMUDEZ, A. C. et al. Sistema de apoio ao controle de informação - SACI. São Carlos, 2005.

BLAIR, D. C. Information retrieval and the philosophy of language. ARIST Annual Review of Information Science and Technology, v. 37, p. 3-50, 2003.

Wittgenstein, Language and Information: "back to the rough ground!" In: INTERNATIONAL CONFERENCE ON CONCEPTIONS OF LIBRARY AND INFORMATION SCIENCES, 5., 2005, Glasgow. Proceedings...Heidelberg: Springer, 2006. p. 1-4. (Lectures Notes Computer Science, v. 3507)

BOTELHO, R.; BELA, R. Sistemas convergentes e interativos de comunicação social. In: SIMPÓSIO DE CIÊNCIAS DA COMUNICAÇÃO NA REGIÃO SUDESTE, 11., 2006, Ribeirão Preto. Resumos... São Paulo, 2006.

BROWN, P. et al. The democratic indexing of images. New Review of Hypermedia and Multimedia, v. 2, n.1, p. 107-120, 1996.

CINTRA, A. M. M. et al. Para entender as linguagens documentárias. São Paulo: Polis, 2002.

LANCASTER, F. W. Indexação e resumos: teoria e prática. Brasília: Briquet de Lemos, 2004.

CAMPOS, M. L. A; GOMES, H. E; MOTTA, D. F. Elaboração de tesauro documentário: tutorial. Disponível em: <www.conexaorio.com/biti/tesauro/> Acesso em 23 mai. 2010. 
CICILLINI, F. M.; FRANCISCO, R. E. B. Gestão institucional: uma aproximação teóricoprática entre sistemas e comunicação. In: CONGRESSO BRASILEIRO DE CIÊNCIAS DA COMUNICAÇÃO, 30., 2007, Santos. Resumos... São Paulo, 2007.

DEODATO, M.; BOTELHO, R. Coordenadoria de comunicação social. São Carlos, 2004. Disponível em: <http://www.comunicacao.ufscar.br/>. Acesso em: fev. 2010.

DOUSA, T. M. Whiter pragmatism in knowledge organization: classical pragmatism vs. neopragmatism as KO metatheories. In: GNOLI, C.; MAZZOCCHI, F.(Eds.) Paradigms and conceptual systems in knowledge organization: proceedings of the Eleventh International ISKO Conference 23-26 February 2010 Rome. Roma: Ergon Verlag, 2010. p. 78-84.

FROHMANN, B. Rules of indexing: a critique of mentalism in information retrieval theory. Journal of documentation. London, v. 46, n. 2, p. 81-101, 1990.

GILCHRIST, Alan. The thesaurus in retrieval. London: Aslib, 1971.

GONZÁLEZ DE GOMEZ, M. N. Da representação do conhecimento ao conhecimento da representação. Ciência da Informação, Brasília, v. 22, n. 3, p. 217-22, 1993.

. Novas fronteiras tecnológicas das ações de informação: questões e abordagens. Ciência da Informação, Brasília, v. 33, n. 1, p. 55-67, 2004.

GRACIOSO, L. de S. Ciência da Informação e a ação comunicativa no cenário Web. In: ENCONTRO NACIONAL DE PESQUISA EM CIÊNCIA DA INFORMAÇÃO, 8., 2007, Salvador. Anais..., Salvador, 2007.

Filosofia da linguagem e Ciência da Informação: jogos de linguagem e ação comunicativa no contexto das ações de informação em tecnologias virtuais. Rio de Janeiro, 2008.175 f. Tese (Doutorado). - Universidade Federal Fluminense, Instituto Brasileiro de Informação em Ciência e Tecnologia, Rio de Janeiro, 2008.

. Pragmatic approach to virtual information action from Wittgenstein. In: GNOLI, C.; MAZZOCCHI, F.(Eds.) Paradigms and conceptual systems in knowledge organization: proceedings of the Eleventh International ISKO Conference 23-26 February 2010 Rome. Roma: Ergon Verlag, 2010. p. 106-114.

GUEDES, R. M.; DIAS, E. W. Indexação social: abordagem conceitual. Revista ACB: Biblioteconomia em Santa Catarina, Florianópolis, v.15, n.1, p. 39-53, jan./jun., 2010.

GUIMARAES, J. A. C.; FERNANDEZ-MOLINA, J. C.; VIDOTTI, S. A. B. G.; FLAMINO, A.; SOUZA, A. S.; CAMARGO, L. S. A.; SILVA, M. S.; RAMALHO, R. A. S. Aspectos éticos de las nuevas tecnologías de la información y la comunicación y su reflejo en la organización del conocimiento. In: GASCÓN, J; BURGUILLOS, F.; PONS, A. (Org.). La dimensión humana de la organización del conocimiento/ The human dimension of knowledge organzation / La dimensió humana de l' organizació del coneixement. Barcelona: Universitat de Barcelona, 2005. p. 177-186.

HABERMAS, J. The theory of communicative action: reason and the rationalization of society. Trad. Thomas McCarthy. Boston: Beacon Press, 1981. v. 1. 
HJORLAND, B. Information retrieval, text composition and semantic. Knowledge Organization., v. 25, n.1-2, 1998.

. Concept theory. Journal of the American Society for Information Science and Technology, v. 60, n. 8, p. 1519-1536, 2009.

. Concepts paradigms and knowledge organization. In: GNOLI, C.; MAZZOCCHI, F.(Eds.) Paradigms and conceptual systems in knowledge organization: proceedings of the Eleventh International ISKO Conference 23-26 February 2010 Rome. Roma: Ergon Verlag, 2010. p. 38-42.

HUTCHINS, W.J. Languages of indexing and classification: a linguistic study of structures and functions. Herts: Peter Peregrinus, 1975.

HUVILA, I. Aesthetic judgments in folksonomies as criteria for organising knowledge. In: GNOLI, C.; MAZZOCCHI, F.(Eds.) Paradigms and conceptual systems in knowledge organization: proceedings of the Eleventh International ISKO Conference 23-26 February 2010 Rome. Roma: Ergon Verlag, 2010. p. 308-315.

KOBASHI, N. Y. Fundamentos semânticos e pragmáticos da construção de instrumentos de representação de informação. Datagramazero - Revista de Ciência da Informação, v.8, n. 6, dez. 2007.

LARA, M. L. G. Informação, informatividade e linguística documentária: alguns paralelos com as reflexões de Hjorland e Capurro. Datagramazero - Revista de Ciência da Informação, v.9, n. 6, ago. 2008.

LYKKE et al. Using semantic components to represent and search domain-specific documents: an evaluation of indexing accuracy and consistency. In: GNOLI, C.;

MAZZOCCHI, F.(Eds.) Paradigms and conceptual systems in knowledge organization: proceedings of the Eleventh International ISKO Conference 23-26 February 2010 Rome. Roma: Ergon Verlag, 2010. p. 276-282.

MAIA, C.; BASSOLINI, M.; BETOLNI, N. Análise da coordenadoria de comunicação social da UFSCar, campus São Carlos: proposta de melhoria no processo de comunicação com base nas teorias de gestão do conhecimento e melhoria de processos. São Carlos, 2008.

MOREIRA, M. P.; MOURA, M. A. Construindo tesauros a partir de tesauros existentes: a experiência do TCI - Tesauro em Ciência da Informação: Datagramazero - Revista de Ciência da Informação, v.7, n. 4, ago. 2006.

MOURA, M. A.; ASSIS, J. Social networks, indexing languages and organization of knowledge: a semiotic approach. In: GNOLI, C.; MAZZOCCHI, F.(Eds.) Paradigms and conceptual systems in knowledge organization: proceedings of the Eleventh International ISKO Conference 23-26 February 2010 Rome. Roma: Ergon Verlag, 2010. p. 291-298. 
NATIONAL INFORMATION STANDARDS ORGANIZATION (2005). ANSI/NISO Z39.19-2003: guidelines for the construction, format, and management of monolingual thesauri. 2005. Disponível em: < www.niso.org/standards/resources/Z39-192005.pdf\#search=\%22z39.19\%22>. Acesso em: 23 de junho de 2010.

NEDOBITY, W. Concepts versus meaning as reflected by the works of W. Wüster and L. Wittgenstein. International Classification, v. 16, n. 1, p.24-26, 1989.

NOVELLINO, M. S. F. A linguagem como meio de representação ou de comunicação da informação. Perspectivas em Ciência da Informação, Belo Horizonte, v. 3, n. 2, p. 137-146, jul./dez. 1998.

PINHO, F. A. Aspectos éticos em representação do conhecimento: em busca do diálogo entre Antonio García Gutiérrez, Michèle Hudon e Clare Beghtol. 2006.123 f. Dissertação (Mestrado) - Faculdade de Filosofia e Ciências, Universidade Estadual Paulista, Marília, 2006.

PRISS, U.; OLD, L. J. Concept neighbourhoods in knowledge organization systems. In: GNOLI, C.; MAZZOCCHI, F.(Eds.) Paradigms and conceptual systems in knowledge organization: proceedings of the Eleventh International ISKO Conference 23-26 February 2010 Rome. Roma: Ergon Verlag, 2010. p. 165-170.

RAFFERTY, P.; HIDDERLEY, R.. Flickr and democratic indexing: dialogic approaches to indexing. Aslib Proceedings, v. 59, n. 4/5, p. 397-410, 2007. Disponível em: < www.emeraldinsight.com/Insight/viewPDF.jsp?Filename=html/Output/Published/EmeraldFul 1TextArticle/Pdf/2760590407.pdf>. Acesso em: 23 de junho de 2010.

SMIRAGLIA, R. P. Perception, KO, and noetic affective social tagging. In: GNOLI, C.; MAZZOCCHI, F.(Eds.) Paradigms and conceptual systems in knowledge organization: proceedings of the Eleventh International ISKO Conference 23-26 February 2010 Rome. Roma: Ergon Verlag, 2010. p. 64-70.

SVENONIUS, Elaine. The intellectual foundation of information organization. Cambridge: MIT Press, 2000.

VAKKARI, P. How specific thesauri and a general thesaurus cover lay persons' vocabularies concerning health, nutrition and social services. In: GNOLI, C.; MAZZOCCHI, F.(Eds.)

Paradigms and conceptual systems in knowledge organization: proceedings of the Eleventh International ISKO Conference 23-26 February 2010 Rome. Roma: Ergon Verlag, 2010. p. $299-307$ 